\title{
The Order Completion Method for Systems of Nonlinear PDEs: Solutions of Initial Value Problems
}

\author{
Jan Harm van der Walt \\ Department of Mathematics and Applied Mathematics, University of Pretoria, Lynwood Road, \\ Pretoria 0002, South Africa \\ Correspondence should be addressed to Jan Harm van der Walt; janharm.vanderwalt@up.ac.za
}

Received 18 July 2012; Revised 11 September 2012; Accepted 16 September 2012

Academic Editor: Svatoslav Stanek

Copyright (C) 2013 Jan Harm van der Walt. This is an open access article distributed under the Creative Commons Attribution License, which permits unrestricted use, distribution, and reproduction in any medium, provided the original work is properly cited.

We present an existence result for generalized solutions of initial value problems obtained through the order completion method. The solutions we obtain satisfy the initial condition in a suitable extended sense, and each such solution may be represented in a canonical way through its generalized partial derivatives as nearly finite normal lower semicontinuous function.

\section{Introduction}

It is a virtual consensus among mathematicians specializing in nonlinear partial differential equations (PDEs) that a general and type independent theory for the existence and basic regularity of generalized solutions of such equations is not possible [1]; see also [2]. Within the setting of the usual linear topological spaces of generalized functions that are customary in the study of PDEs, this may perhaps turn out to be the case. Here we may point out two possible reasons for the failure of the mentioned customary spaces of generalized functions to contain solutions of large classes of linear and nonlinear PDEs.

Firstly, these spaces typically fail to contain sufficiently singular objects. Indeed, the Sobolev spaces have been so successful in the study of PDEs exactly because, in some cases, they lead to rather regular, in fact even smooth, generalized solutions of PDEs. On the other hand, singularities which may occur in the solutions of nonlinear PDEs may be rather arbitrary. We may recall that even in the case of analytic nonlinear PDEs, the Cauchy-Kovalevskaia Theorem [3] guarantees the existence of an analytic solution only on a neighborhood of a given noncharacteristic analytic hypersurface. As such, any solution which is defined on the whole domain of definition of a given analytic system of nonlinear PDEs, see, for instance [4], will in general admit singularities. In the simplest case of an analytic function with an essential singularity at a single point, the Great Picard Theorem states that the function will attain every complex value, with possibly one exception, in every neighborhood of the singularity.

This brings us to the second reason for the failure of usual spaces of generalized functions to contain solutions of large classes of systems of nonlinear PDEs. Namely, the generalized functions that are the elements of these spaces are typically defined in terms of certain growth conditions. This is true, for instance, of the Sobolev spaces, the elements of which must be locally integrable, and the Colombeau algebras of generalized functions [5], where the generalized functions are required to satisfy certain polynomial type growth conditions near singularities. In view of our remarks above concerning the existence of solutions of analytic systems of nonlinear PDEs, the deficiency of such growth conditions is clear. Indeed, an analytic function which has an essential singularity at just one point may grow faster than any polynomial near the singularity and may therefore also fail to be locally integrable on any neighborhood of that singularity.

However, and in contradistinction with the perviously mentioned insufficiency of the customary functional analytic methods, the order completion method, published in the 1994 monograph [6], delivers generalized solutions of a large class of systems of continuous nonlinear PDEs. These solutions are constructed as the elements of the Dedekind order completion of suitable spaces of piecewise smooth 
functions. Furthermore, the solutions obtained in this way have been shown to satisfy a basic blanket regularity in the sense that the solutions may be assimilated with usual Hausdorff continuous interval valued functions [7].

Recently, see [8-10], the mentioned order completion method was reformulated and enriched by introducing suitable uniform convergence spaces. This has led to a significant improvement in the regularity of the generalized solutions obtained, as well as significant new insight into the structure of the solutions.

In this paper, we show how the techniques developed in [10] may be adapted in order to also incorporate initial and/or boundary values that may be associated with a given system of nonlinear PDEs. As it turns out, in order to incorporate such addition conditions into the theory, the methods that apply to the free problem need only be modified slightly. This state of affairs should be compared with the usual linear functional analytic techniques for solving linear and nonlinear PDEs, where the presence of initial and/or boundary values often leads to significant complications, which typically require entirely new methods. In this way we come to appreciate yet another advantage of solving nonlinear PDEs by the methods introduced in [10]. Namely, initial and boundary value problems are solved by essentially the same techniques that apply to the free problem.

The paper is organized as follows. In Section 2 we recall some basic concepts relating to the spaces of normal lower semicontinuous functions upon which the spaces of generalized functions are constructed in Section 3. The existence of generalized solutions of a large class of initial value problems is presented in Section 4, where we also discuss the structure and regularity of the solutions.

\section{Normal Lower Semicontinuous Functions}

In this section we recall some basic facts concerning spaces of normal lower semicontinuous functions upon which the spaces of generalized functions are constructed. In particular, the spaces of generalized functions are constructed as the completions of suitable uniform convergence spaces, the elements of which are normal lower semicontinuous functions. In order to make the exposition as self-contained as possible, we also include a brief account of the spaces introduced in [8-10].

In this regard, let $\Omega$ be an open subset of $\mathbb{R}^{k}$, and denote by $\mathscr{A}(\Omega)$ the set of extended real valued functions on $\Omega$. That is, $\mathscr{A}(\Omega)=\{u: \Omega \rightarrow \overline{\mathbb{R}}\}$, where $\overline{\mathbb{R}}=\mathbb{R} \cup\{ \pm \infty\}$ is the extended real line. The lower and upper Baire operators $I$ : $\mathscr{A}(\Omega) \rightarrow \mathscr{A}(\Omega)$ and $S: \mathscr{A}(\Omega) \rightarrow \mathscr{A}(\Omega)$ are defined through

$$
I(u): \Omega \ni x \longmapsto \sup \left\{\inf \{u(y): y \in V\}: V \in \mathscr{V}_{x}\right\} \in \overline{\mathbb{R}}
$$

$$
S(u): \Omega \ni x \longmapsto \inf \left\{\sup \{u(y): y \in V\}: V \in \mathscr{V}_{x}\right\} \in \overline{\mathbb{R}},
$$

respectively, where $\mathscr{V}_{x}$ denotes the neighborhood filter at $x \epsilon$ $\Omega$, see [11] or [12] for a recent presentation. The mappings (1) and (2) satisfy

$$
\begin{aligned}
& \forall u \in \mathscr{A}(\Omega): \\
& \quad I(u) \leq u \leq S(u) .
\end{aligned}
$$

Furthermore, the operators $I, S$ and their compositions are idempotent and monotone with respect to the pointwise order on $\mathscr{A}(\Omega)$. That is,

$$
\begin{aligned}
& \forall u \in \mathscr{A}(\Omega): \\
& \text { (1) } I(I(u))=I(u), \\
& \text { (2) } S(S(u))=S(u), \\
& \text { (3) }(I \circ S)((I \circ S)(u))=(I \circ S)(u),
\end{aligned}
$$

and

$$
\begin{aligned}
& \forall u, v \in \mathscr{A}(\Omega): \\
& u \leq v \Longrightarrow\left(\begin{array}{l}
(1) \quad I(u) \leq I(v) \\
(2) S(u) \leq S(v) \\
(3)(I \circ S)(u) \leq(I \circ S)(v)
\end{array}\right) .
\end{aligned}
$$

A function $u \in \mathscr{A}(\Omega)$ is normal lower semicontinuous at $x \in \Omega$ whenever

$$
(I \circ S)(u)(x)=u(x),
$$

while $u$ is normal lower semicontinuous on $\Omega$ provided it is normal lower semicontinuous at every point $x \in \Omega$; see [12, 13]. A normal lower semicontinuous function is called nearly finite whenever

$$
\{x \in \Omega: u(x) \in \mathbb{R}\} \text { is open and dense in } \Omega .
$$

The set of nearly finite normal lower semicontinuous functions on $\Omega$ is denoted by $\mathcal{N} \mathscr{L}(\Omega)$. Clearly, every continuous, real valued function on $\Omega$ is nearly finite and normal lower semicontinuous, so that we have the inclusion

$$
\mathscr{C}^{0}(\Omega) \subseteq \mathscr{N} \mathscr{L}(\Omega) .
$$

Conversely, each function $u \in \mathscr{N} \mathscr{L}(\Omega)$ is continuous on a residual set. That is,

$$
\begin{aligned}
& \forall u \in \mathscr{N} \mathscr{L}(\Omega): \\
& \exists B \subset \Omega \text { of first Baire category: } \\
& \quad x \in \Omega \backslash B \Longrightarrow u \text { is continuous at } x .
\end{aligned}
$$

The following useful property of continuous functions extends to $\mathscr{N} \mathscr{L}(\Omega)$ :

$$
\begin{aligned}
& \forall u, v \in \mathcal{N L}(\Omega): \\
& \forall D \subseteq \Omega \text { dense : }
\end{aligned}
$$$$
(\forall x \in D \text { : }
$$

$$
u(x) \leq v(x)) \Longrightarrow u \leq v .
$$


With respect to the pointwise order

$$
u \leq v \Longleftrightarrow\left(\begin{array}{c}
\forall x \in \Omega: \\
u(x) \leq v(x)
\end{array}\right)
$$

the set $\mathscr{N} \mathscr{L}(\Omega)$ is a Dedekind complete lattice. In particular, the supremum and infimum of a set $\mathscr{A} \subset \mathcal{N} \mathscr{L}(\Omega)$ is given by

$$
\begin{aligned}
\inf \mathscr{A} & =(I \circ S)(\varphi), \\
\sup \mathscr{A} & =(I \circ S)(\psi),
\end{aligned}
$$

respectively, where $\varphi: \Omega \ni x \mapsto \inf \{u(x): u \in \mathscr{A}\}$ and $\psi: \Omega \ni x \mapsto \sup \{u(x): u \in \mathscr{A}\}$. Furthermore, the lattice $\mathscr{N} \mathscr{L}(\Omega)$ is fully distributive. That is,

$$
\begin{aligned}
& \forall v \in \mathscr{N} \mathscr{L}(\Omega): \\
& \forall \mathscr{A} \subset \mathscr{N} \mathscr{L}(\Omega): \\
& \quad u_{0}=\sup \mathscr{A} \Longrightarrow \sup \{\inf \{u, v\}: u \in \mathscr{A}\}=\inf \left\{u_{0}, v\right\} .
\end{aligned}
$$

A useful characterization of order bounded sets in terms of pointwise bounded sets is given as follows. If a set $\mathscr{A} \subset$ $\mathscr{N} \mathscr{L}(\Omega)$ satisfies

$$
\begin{aligned}
& \exists B \subset \Omega \text { of first Baire category: } \\
& \forall x \in \Omega \backslash B: \\
& \quad \sup \{u(x): u \in \mathscr{A}\}<\infty,
\end{aligned}
$$

then

$$
\begin{aligned}
& \exists u_{0} \in \mathscr{N} \mathscr{L}(\Omega): \\
& \forall u \in \mathscr{A}: \\
& u \leq u_{0} .
\end{aligned}
$$

The dual statement for sets bounded from below also holds.

For $m \in \mathbb{N} \cup\{0\}$, we consider the set

$$
\begin{aligned}
& \mathscr{M}_{\mathscr{L}^{m}(\Omega)} \\
& =\{u \in \mathscr{N} \mathscr{L}(\Omega) \mid \\
& \exists \Gamma \subset \Omega \text { closed nowhere dense : } \\
& \left.\quad u \in \mathscr{C}^{m}(\Omega \backslash \Gamma)\right\} .
\end{aligned}
$$

Each of the spaces $\mathscr{M} \mathscr{L}^{m}(\Omega)$ is a sublattice of $\mathscr{N} \mathscr{L}(\Omega)$; see [14]. In particular, $\mathscr{M} \mathscr{L}^{0}(\Omega)$ is $\sigma$-order dense in $\mathscr{N} \mathscr{L}(\Omega)$. That is, for each $u \in \mathscr{N} \mathscr{L}(\Omega)$ we have

$$
\begin{aligned}
& \exists\left(\lambda_{n}\right),\left(\mu_{n}\right) \subset \mathscr{M L}^{0}(\Omega): \\
& \quad \text { (1) } \lambda_{n} \leq \lambda_{n+1} \leq u \leq \mu_{n+1} \leq \mu_{n}, \quad n \in \mathbb{N}, \\
& \quad \text { (2) } \sup \left\{\lambda_{n}: n \in \mathbb{N}\right\}=u=\inf \left\{\mu_{n}: n \in \mathbb{N}\right\} .
\end{aligned}
$$

The spaces of generalized functions introduced in [10] are constructed as the completions of suitable uniform convergence spaces. In this regard, a uniform convergence structure is defined on $\mathscr{M} \mathscr{L}^{0}(\Omega)$ in the following way.
Definition 1. Let $\Sigma$ consist of all nonempty order intervals in $\mathscr{M} \mathscr{L}^{0}(\Omega)$. Let $\mathscr{J}_{o}$ denote the family of filters on $\mathscr{M} \mathscr{L}^{0}(\Omega) \times$ $\mathscr{M} \mathscr{L}^{0}(\Omega)$ that satisfy the following. There exists $k \in \mathbb{N}$ such that

$$
\begin{aligned}
& \forall j=1, \ldots, k: \\
& \exists \Sigma_{i}=\left(I_{n}^{j}\right) \subseteq \Sigma: \\
& \exists u_{j} \in \mathcal{N} \mathscr{L}(\Omega): \\
& \text { (1) } I_{n+1}^{j} \subseteq I_{n}^{j}, \quad n \in \mathbb{N}, \\
& \text { (2) } \sup \left\{\inf I_{n}^{j}: n \in \mathbb{N}\right\}=u_{j}=\inf \left\{\sup I_{n}^{j}: n \in \mathbb{N}\right\}, \\
& \text { (3) }\left(\left[\Sigma_{1}\right] \times\left[\Sigma_{1}\right]\right) \cap \cdots \cap\left(\left[\Sigma_{k}\right] \times\left[\Sigma_{k}\right]\right) \subseteq \mathscr{U} .
\end{aligned}
$$

The uniform convergence structure $\mathscr{J}_{o}$ is first countable and uniformly Hausdorff. Furthermore, a filter $\mathscr{F}$ on $\mathscr{M}^{0}(\Omega)$ converges to $u \in \mathscr{M} \mathscr{L}^{0}(\Omega)$ with respect to $\mathscr{J}_{o}$ if and only if

$$
\begin{aligned}
& \exists\left(\lambda_{n}\right),\left(\mu_{n}\right) \subset \mathscr{M} \mathscr{L}^{0}(\Omega): \\
& \text { (1) } n \in \mathbb{N} \Longrightarrow \lambda_{n} \leq \lambda_{n+1} \leq \mu_{n+1} \leq \mu_{n}, \\
& \text { (2) } \sup \left\{\lambda_{n}: n \in \mathbb{N}\right\}=u=\inf \left\{\mu_{n}: n \in \mathbb{N}\right\}, \\
& \text { (3) }\left[\left\{\left[\lambda_{n}, \mu_{n}\right]: n \in \mathbb{N}\right\}\right] \subseteq \mathscr{F} .
\end{aligned}
$$

The completion of the space $\mathscr{M} \mathscr{L}^{0}(\Omega)$ with respect to the uniform convergence structure $\mathscr{J}_{0}$ may be represented as the set $\mathscr{N} \mathscr{L}(\Omega)$, equipped with the appropriate uniform convergence structure. This completion result follows essentially as an application of the order completeness of $\mathscr{N} \mathscr{L}(\Omega)$ and the approximation property (18). The correct uniform convergence structure on $\mathscr{N} \mathscr{L}(\Omega)$ is defined as follows.

Definition 2. A filter $\mathcal{U}$ on $\mathscr{N} \mathscr{L}(\Omega) \times \mathscr{N} \mathscr{L}(\Omega)$ belongs to the family $\mathscr{J}_{o}^{\sharp}$ whenever, for some positive integer $k$, we have the following:

$$
\begin{aligned}
& \forall i=1, \ldots, k: \\
& \exists\left(\lambda_{n}^{i}\right),\left(\mu_{n}^{i}\right) \subset \mathscr{M} \mathscr{L}^{0}(\Omega): \\
& \exists u^{i} \in \mathcal{N} \mathscr{L}(\Omega): \\
& \quad \text { (1) } \lambda_{n}^{i} \leq \lambda_{n+1}^{i} \leq \mu_{n+1}^{i} \leq \mu_{n}^{i}, \quad n \in \mathbb{N}, \\
& \text { (2) } \sup \left\{\lambda_{n}^{i}: n \in \mathbb{N}\right\}=u^{i}=\inf \left\{\mu_{n}^{i}: n \in \mathbb{N}\right\}, \\
& \text { (3) } \bigcap_{i=1}^{k}\left(\left(\left[\Sigma^{i}\right] \times\left[\Sigma^{i}\right]\right) \cap\left(\left[u^{i}\right] \times\left[u^{i}\right]\right)\right) \subseteq \mathscr{U} .
\end{aligned}
$$

Here $\Sigma^{i}=\left\{I_{n}^{i}: n \in \mathbb{N}\right\}$ with $I_{n}^{i}=\left\{u \in \mathscr{M} \mathscr{L}^{0}: \lambda_{n}^{i} \leq u \leq \mu_{n}^{i}\right\}$.

For $m \geq 1$ the usual linear partial differential operators $D^{\alpha}: \mathscr{C}^{m}(\Omega) \rightarrow \mathscr{C}^{0}(\Omega),|\alpha| \leq m$ extend uniquely to mappings

$$
\mathscr{D}^{\alpha}: \mathscr{M} \mathscr{L}^{m}(\Omega) \longrightarrow \mathscr{M} \mathscr{L}^{0}(\Omega), \quad|\alpha| \leq m,
$$


which may be defined as

$$
\mathscr{D}^{\alpha}: \mathscr{M} \mathscr{L}^{m}(\Omega) \ni u \longmapsto(I \circ S)\left(D^{\alpha} u\right) \in \mathscr{M} \mathscr{L}^{0}(\Omega), \quad|\alpha| \leq m .
$$

The space $\mathscr{M} \mathscr{L}^{m}(\Omega)$ is equipped with the initial uniform convergence structure $\mathscr{J}_{m}$ with respect to the family of mappings (22). That is,

$$
\mathcal{U} \in \mathscr{J}_{m} \Longleftrightarrow\left(\begin{array}{l}
\forall|\alpha| \leq m: \\
\quad\left(\mathscr{D}^{\alpha} \times \mathscr{D}^{\alpha}\right)(\mathscr{U}) \in \mathscr{J}_{o}
\end{array}\right) .
$$

Clearly $\mathscr{J}_{m}$ makes each of the mappings (22) uniformly continuous. In fact, it is the coarsest uniform convergence structure with respect to which each of the mappings (22) is uniformly continuous. Since the family of mappings (22) is countable, it follows from the first countability of $\mathscr{J}_{0}$ that the uniform convergence structure $\mathscr{J}_{m}$ is also first countable. Furthermore, the family of mappings (22) separates the points of $\mathscr{M} \mathscr{L}^{m}(\Omega)$, that is:

$$
\begin{aligned}
& \forall u, v \in \mathscr{M} \mathscr{L}^{m}(\Omega): \\
& \exists|\alpha| \leq m: \\
& \mathscr{D}^{\alpha} u \neq \mathscr{D}^{\alpha} v,
\end{aligned}
$$

so that the uniform convergence structure $\mathscr{J}_{m}$ is uniformly Hausdorff. As such, we may construct its completion, which we denote by $\mathcal{N}^{m}(\Omega)$. This notation is due to the fact that, as we will shortly see, we may identify the completion of $\mathscr{M} \mathscr{L}^{m}(\Omega)$ in a canonical way with a subspace of $\mathscr{N} \mathscr{L}(\Omega)^{M}$, for a suitable integer $M$. Indeed, see [14], the mapping

$$
\text { D : } \mathscr{M} \mathscr{L}^{m}(\Omega) \ni u \longmapsto\left(\mathscr{D}^{\alpha} u\right)_{|\alpha| \leq m} \in \mathscr{M} \mathscr{L}^{0}(\Omega)^{M} \text {, }
$$

with $\mathscr{M} \mathscr{L}^{0}(\Omega)^{M}$ equipped with the product uniform convergence structure, is a uniformly continuous embedding. As such, it may be extended in a unique way to an injective uniformly continuous mapping

$$
\mathrm{D}^{\sharp}: \mathcal{N} \mathscr{L}^{m}(\Omega) \ni u^{\sharp} \longmapsto\left(\mathscr{D}^{\alpha \sharp} u^{\sharp}\right)_{|\alpha| \leq m} \in \mathcal{N} \mathscr{L}(\Omega)^{M} \text {. }
$$

Here the mappings

$$
\mathscr{D}^{\alpha \sharp}: \mathscr{N} \mathscr{L}^{m}(\Omega) \longrightarrow \mathscr{N} \mathscr{L}(\Omega), \quad|\alpha| \leq m
$$

are the unique uniformly continuous extensions of the mappings (22).

\section{Spaces of Generalized Functions}

The space of generalized functions $\mathcal{N}^{m}(\Omega)$ was shown in [10] to contain generalized solutions of a large class of systems of nonlinear PDEs. However, as mentioned in Section 1, this existence result does not take into account any initial and/or boundary values that may be associated with a given system of nonlinear PDEs. In order to also incorporate such additional conditions, we need only modify the construction of the space $\mathscr{N} \mathscr{L}^{m}(\Omega)$ slightly.
In this regard, consider a system of $K$ nonlinear PDEs:

$$
D_{t}^{m} \mathbf{u}(t, y)=\mathbf{G}\left(t, y, \ldots, D_{y}^{q} D_{t}^{p} u_{i}(t, y), \ldots\right)
$$

with $t \in \mathbb{R}, y \in \mathbb{R}^{k-1}, m \geq 1,0 \leq p<m, q \in \mathbb{N}^{k-1},|q|+p \leq m$ and with the Cauchy data

$$
D_{t}^{p} \mathbf{u}\left(t_{0}, y\right)=\mathbf{g}_{p}(y), \quad 0 \leq p<m,\left(t_{0}, y\right) \in S
$$

on the hyperplane

$$
S=\left\{\left(t_{0}, y\right): y \in \mathbb{R}^{k-1}\right\}
$$

We assume that the initial data (30) satisfies

$$
\forall 0 \leq p<m: \mathbf{g}_{p} \in \mathscr{C}^{m-p}\left(\mathbb{R}^{k-1}\right)^{K}
$$

It follows immediately from the results presented in [10] that the system of nonlinear PDEs (29) admits a generalized solution in $\mathscr{N} \mathscr{L}^{m}\left(\mathbb{R}^{k}\right)^{K}$. However, such a solution may fail to satisfy the initial condition (30) in any suitable extended sense.

In order to incorporate the initial condition (30) into our solution method, we introduce the following spaces of functions. Denote by $\mathscr{M}_{\mathscr{L}_{\mathbf{g}}^{m}}(\Omega)$ the set

$$
\begin{aligned}
& \mathscr{M}_{\mathbf{g}}^{m}(\Omega) \\
& =\left\{\begin{array}{ll}
\mathbf{u} \in \mathscr{M} \mathscr{L}^{m}(\Omega)^{K} & \begin{array}{l}
\forall i=1, \ldots, K: \\
\forall 0 \leq p<m: \\
\forall q \in \mathbb{N}^{k-1}, 0 \leq|q|+p \leq m: \\
\text { (1) } \mathscr{D}_{y t}^{q p} u_{i}\left(y, t_{0}\right)=D^{q} g_{p, i}(y), \quad y \in \mathbb{R}^{k-1} \\
\text { (2) } \mathscr{D}_{y t}^{q p} u_{i} \text { is continuous at }\left(y, t_{0}\right)
\end{array}
\end{array}\right\},
\end{aligned}
$$

where $\Omega=\mathbb{R}^{k-1} \times \mathbb{R}$. For each $i=1, \ldots, K$, every $0 \leq p<m$ and each $q \in \mathbb{N}^{k-1}$ such that $0 \leq|q|+p \leq m$, we consider the space $\mathscr{M} \mathscr{L}_{i, q, p}^{0}(\Omega)$, which is defined through

$$
\begin{aligned}
& \mathscr{M} \mathscr{L}_{i, q, p}^{0}(\Omega) \\
& =\left\{\begin{array}{l|l}
u \in \mathscr{M} \mathscr{L}^{0}(\Omega) & \begin{array}{l}
\forall y \in \mathbb{R}^{k-1}: \\
\text { (1) } u\left(y, t_{0}\right)=D^{q} g_{p, i}(y) \\
(2) u \text { is continuous at }\left(y, t_{0}\right)
\end{array}
\end{array}\right\} .
\end{aligned}
$$

Clearly, for every $0 \leq p<m$, and $p \in \mathbb{N}^{k-1}$ such that $0 \leq$ $|q|+p \leq m$, and each $i=1, \ldots, K$ we may define the partial differential operators

$$
\mathscr{D}_{i, y t}^{q p}: \mathscr{M} \mathscr{L}_{\mathbf{g}}^{m}(\Omega) \longrightarrow \mathscr{M}_{i, q, p}^{0}(\Omega),
$$

as in Section 2 through

$$
\mathscr{D}_{i, y t}^{q p} \mathbf{u}=(I \circ S)\left(D_{y t}^{q p} u_{i}\right)
$$

The partial differential operator $\mathscr{D}_{i, t}^{m}$ is defined in a similar way, namely, as

$$
\mathscr{D}_{i, t}^{m}: \mathscr{M}_{\mathbf{g}}^{m}(\Omega) \ni \mathbf{u} \longmapsto(I \circ S)\left(D_{t}^{m} u_{i}\right) \in \mathscr{M}^{0}(\Omega) .
$$


The method for constructing generalized solutions of the initial value problem (29) to (30) presented here is essentially the same as that used in the case of arbitrary systems of nonlinear PDEs, which is developed in [10]. In particular, generalized solutions are constructed as elements of the completion of the space $\mathscr{M L}_{\mathbf{g}}^{m}(\Omega)$, equipped with a suitable uniform convergence structure. In this regard, we introduce the following uniform convergence structure on $\mathscr{M} \mathscr{L}_{i, q, p}^{0}(\Omega)$.

Definition 3. Let $\Sigma$ consist of all nonempty order intervals in $\mathscr{M} \mathscr{L}_{i, q, p}^{0}(\Omega)$. Let $\mathscr{J}_{i, q, p}$ denote the family of filters on $\mathscr{M} \mathscr{L}_{i, q, p}^{0}(\Omega) \times \mathscr{M}_{i, q, p}^{0}(\Omega)$ that satisfy the following. There exists $k \in \mathbb{N}$ such that

$$
\begin{aligned}
& \forall j=1, \ldots, k: \\
& \exists \Sigma_{j}=\left(I_{n}^{j}\right) \subseteq \Sigma: \\
& \exists u_{j} \in \mathscr{N} \mathscr{L}(\Omega):
\end{aligned}
$$

(1) $I_{n+1}^{j} \subseteq I_{n}^{j}, \quad n \in \mathbb{N}$,

(2) $\sup \left\{\inf I_{n}^{j}: n \in \mathbb{N}\right\}=u_{j}=\inf \left\{\sup I_{n}^{j}: n \in \mathbb{N}\right\}$,

(3) $\left(\left[\Sigma_{1}\right] \times\left[\Sigma_{1}\right]\right) \cap \cdots \cap\left(\left[\Sigma_{k}\right] \times\left[\Sigma_{k}\right]\right) \subseteq \mathscr{U}$.

Proposition 4. The family of filters $\mathscr{F}_{i, q, p}$ on $\mathscr{M}_{\mathscr{L}_{i, q, p}^{0}}^{0}(\Omega) \times$ $\mathscr{M} \mathscr{L}_{i, q, p}^{0}(\Omega)$ is a Hausdorff uniform convergence structure.

Furthermore, a filter $\mathscr{F}$ on $\mathscr{M} \mathscr{L}_{i, q, p}^{0}(\Omega)$ converges to $u \in$ $\mathscr{M} \mathscr{L}_{i, q, p}^{0}(\Omega)$ if and only if there exists a family $\Sigma_{\mathscr{F}}=\left(I_{n}\right)$ of nonempty order intervals on $\mathscr{M L}_{i, q, p}^{0}(\Omega)$ such that

$$
\begin{aligned}
& \text { (1) } I_{n+1} \subseteq I_{n}, \quad n \in \mathbb{N} \text {, } \\
& \text { (2) } \forall V \subseteq \Omega \text { nonempty and open : }
\end{aligned}
$$

$$
\bigcap_{n \in \mathbb{N}} I_{n \mid V}=\{u\}_{\mid V}
$$

and $\left[\Sigma_{\mathscr{F}}\right] \subseteq \mathscr{F}$.

Proof. The first four axioms of the definition of a uniform convergence structure [15] are clearly fulfilled, so it remains to verify

$$
\begin{aligned}
& \forall \mathscr{U}, \mathscr{V} \in \mathscr{J}_{0}: \\
& \quad \mathcal{U} \circ \mathscr{V} \text { exists } \Longrightarrow \mathscr{U} \circ \mathscr{V} \in \mathscr{J}_{0} .
\end{aligned}
$$

In this regard, take any $\mathscr{U}, \mathscr{V} \in \mathscr{J}_{0}$ such that $\mathscr{U} \circ \mathscr{V}$ exists, and let $\Sigma_{1}, \ldots, \Sigma_{k}$ and $\Sigma_{1}^{\prime}, \ldots, \Sigma_{l}^{\prime}$ be the collections of order intervals associated with $\mathscr{U}$ and $\mathscr{V}$, respectively, through Definition 3. Set

$$
\Phi=\left\{(l, j):\left[\Sigma_{l}\right] \circ\left[\Sigma_{j}^{\prime}\right] \text { exists }\right\} .
$$

Then, by [15, Lemma 2.1.1]

$$
\mathcal{U} \circ \mathscr{V} \supseteq \bigcap\left\{\left(\left[\Sigma_{l}\right] \times\left[\Sigma_{l}\right]\right) \circ\left(\left[\Sigma_{j}\right] \times\left[\Sigma_{j}\right]\right):(l, j) \in \Phi\right\} .
$$

Now $(l, j) \in \Phi$ if and only if

$$
\forall m, n \in \mathbb{N}: I_{m}^{l} \cap I_{n}^{j} \neq \emptyset .
$$

For any $(l, j) \in \Phi$, set $\Sigma_{l, j}=\left(I_{n}^{l, j}\right)$ where, for each $n \in \mathbb{N}$

$$
I_{n}^{l, j}=\left[\inf \left(I_{n}^{l}\right) \wedge \inf \left(I_{n}^{j}\right), \sup \left(I_{n}^{l}\right) \vee \sup \left(I_{n}^{j}\right)\right] .
$$

Now, using (42), we find

$$
\begin{aligned}
\mathcal{U} \circ \mathscr{V} & \supseteq \bigcap\left\{\left[\Sigma_{l}\right] \times\left[\Sigma_{j}\right]:(l, j) \in \Phi\right\} \\
& \supseteq \bigcap\left\{\left[\Sigma_{l, j}\right] \times\left[\Sigma_{l, j}\right]:(l, j) \in \Phi\right\} .
\end{aligned}
$$

Since $\mathscr{M} \mathscr{L}^{0}(\Omega)$ is fully distributive, the conditions in (38) follow by Lemma 5 .

The second part of the proposition follows by the same arguments used in the proof of [8, Theorem 8].

The proof of Proposition 4 relies on the following.

Lemma 5. The set $\mathscr{M} \mathscr{L}_{i, q, p}^{0}(\Omega)$ is a lattice with respect to the pointwise order.

Proof. Consider functions $u, v \in \mathscr{M L}_{i, q, p}^{0}(\Omega)$, and set $w=$ $\sup \{u, v\} \in \mathscr{M} \mathscr{L}^{0}(\Omega)$. In view of (13) it follows that $w(x)=$ $(I \circ S)(\varphi)(x), x \in \Omega$ where $\varphi(x)=\sup \{u(x), v(x)\}, x \in \Omega$. Assume that

$$
\exists y_{0} \in \mathbb{R}^{k}, a \in \mathbb{R}: w\left(y_{0}, t_{0}\right)>a>D^{q} g_{p, i}\left(y_{0}\right) .
$$

It then follows that $S(\varphi)\left(y_{0}, t_{0}\right)>a>D^{q} g_{p, i}\left(y_{0}\right)$. Therefore

$$
\begin{aligned}
& \forall \delta>0: \\
& \exists\left(y_{\delta}, t_{\delta}\right) \in B_{\delta}\left(y_{0}, t_{0}\right): \\
& \quad \varphi\left(y_{\delta}, t_{\delta}\right)>a>D^{q} g_{p, i}\left(y_{0}\right)
\end{aligned}
$$

so that we obtain a sequence $\left(y_{n}, t_{n}\right)$ in $\Omega$ which converges to $\left(y_{0}, t_{0}\right)$ and satisfies

$$
\forall n \in \mathbb{N}: u\left(y_{n}, t_{n}\right)>a>D^{q} g_{i, p}\left(y_{0}\right)=u\left(y_{0}, t_{0}\right),
$$

or

$$
\forall n \in \mathbb{N}: v\left(y_{n}, t_{n}\right)>a>D^{q} g_{i, p}\left(y_{0}\right)=v\left(y_{0}, t_{0}\right) .
$$

But both $u$ and $v$ are continuous at $\left(y, t_{0}\right)$ for each $y \in \mathbb{R}^{k}$, which contradicts (48) and (49). Hence (46) cannot hold, so that $w\left(y, t_{0}\right)=D^{q} g_{i, p}(y)$ for each $y \in \mathbb{R}^{k-1}$. Furthermore, since both $u$ and $v$ are continuous at $\left(y, t_{0}\right)$ for each $y \in \mathbb{R}^{k-1}$, it follows that $\varphi$ is continuous at each of these points. As such, $\varphi$ is normal lower semicontinuous at each point $\left(y, t_{0}\right)$ so that we have

$$
\begin{aligned}
w\left(y, t_{0}\right) & =(I \circ S)(\varphi)\left(y, t_{0}\right)=\varphi\left(y, t_{0}\right) \\
& =D^{q} g_{i, p}\left(y, t_{0}\right), \quad y \in \mathbb{R}^{k-1} .
\end{aligned}
$$


In the same way, we see that $w$ is upper semicontinuous at every point $\left(y, t_{0}\right)$. Therefore $w$ is continuous at $\left(y, t_{0}\right)$ for every $y \in \mathbb{R}^{k-1}$ so that $w \in \mathscr{M} \mathscr{L}_{i, q, p}^{0}(\Omega)$.

The existence of the infimum of $u$ and $v$ follows in the same way.

The completion of $\mathscr{M}_{i, q, p}^{0}(\Omega)$ may be represented as a suitable space of nearly finite normal lower semicontinuous functions. In particular, consider the space

$$
\begin{gathered}
\mathscr{N}_{i, q, p}(\Omega) \\
=\{u \in \mathscr{N} \mathscr{L}(\Omega) \\
\mid \exists \lambda, \mu \in \mathscr{M} \mathscr{L}_{i, q, p}^{0}(\Omega): \\
\lambda \leq u \leq \mu\} .
\end{gathered}
$$

Note that $\mathscr{M}_{\mathscr{L}_{i, q, p}^{0}}^{0}(\Omega) \subset \mathcal{N} \mathscr{L}_{i, q, p}(\Omega)$. As such, in order to show that $\mathcal{N} \mathscr{L}_{i, q, p}(\Omega)$ is the completion of $\mathscr{M L}_{i, q, p}^{0}(\Omega)$, we must introduce a Hausdorff uniform convergence structure $\mathscr{L}_{i, q, p}^{\sharp}$ on $\mathcal{N}_{\mathscr{L}_{i, q, p}}(\Omega)$ in such a way that the following conditions are satisfied.

(1) $\mathcal{N}_{\mathscr{L}_{i, q, p}}(\Omega)$ is complete with respect to $\mathscr{J}_{i, q, p}^{\sharp}$.

(2) $\mathscr{N}_{i, q, p}(\Omega)$ contains $\mathscr{M} \mathscr{L}_{i, q, p}^{0}(\Omega)$ as a dense subspace.

(3) If $Y$ is a complete, Hausdorff uniform convergence space, then any uniformly continuous mapping $\varphi$ : $\mathscr{M} \mathscr{L}_{i, q, p}^{0}(\Omega) \rightarrow Y$ extends in a unique way to a uniformly continuous mapping $\varphi^{\sharp}: \mathscr{N}_{i, q, p}(\Omega) \rightarrow$ $Y$.

The appropriate definition of the uniform convergence structure on $\mathcal{N} \mathscr{L}_{i, q, p}(\Omega)$ is similar to Definition 2 .

Definition 6. Let $\mathscr{J}_{i, q, p}^{\sharp}$ denote the family of filters on $\mathscr{N}_{i, q, p}(\Omega) \times \mathscr{N}_{i, q, p}(\Omega)$ that satisfy the following. There exists $k \in \mathbb{N}$ such that

$$
\begin{aligned}
& \forall j=1, \ldots, k: \\
& \exists\left(\lambda_{n}^{j}\right),\left(\mu_{n}^{j}\right) \subseteq \mathscr{M} \mathscr{L}_{i, q, p}^{0}(\Omega): \\
& \exists u_{j} \in \mathcal{N} \mathscr{L}_{i, q, p}(\Omega): \\
& \quad \text { (1) } \lambda_{n}^{j} \leq \lambda_{n+1}^{j} \leq \mu_{n+1}^{j} \leq \mu_{n}^{j}, \quad n \in \mathbb{N} \\
& \quad \text { (2) } \bigcap_{j=1}^{k}\left(\left(\left[\Sigma_{j}\right] \times\left[\Sigma_{j}\right]\right) \cap\left(\left[u_{j}\right] \times\left[u_{j}\right]\right)\right) \subseteq \mathscr{U},
\end{aligned}
$$

where each $u_{j} \in \mathscr{N}_{i, q, p}(\Omega)$ satisfies $u_{j}=\sup \left\{\lambda_{n}^{j}: n \in \mathbb{N}\right\}=$ $\inf \left\{\mu_{n}^{j}: n \in \mathbb{N}\right\}$, and $\Sigma_{j}=\left\{I_{n}^{j}: n \in \mathbb{N}\right\}$ with

$$
I_{n}^{j}=\left\{u \in \mathscr{M}_{i, q, p}(\Omega): \lambda_{n}^{j} \leq u \leq \mu_{n}^{j}\right\} .
$$

That the family of filters $\mathscr{J}_{i, q, p}^{\sharp}$ does indeed constitute a Hausdorff uniform convergence structure on $\mathscr{N}_{i, q, p}(\Omega)$ can easily be seen. Indeed, $\mathscr{F}_{i, q, p}^{\sharp}$ is nothing but the uniform convergence structure associated with the following Hausdorff convergence structure through [15, Proposition 2.1.7]. A filter $\mathscr{F}$ on $\mathscr{N}_{\mathscr{L}_{i, q, p}}(\Omega)$ converges to $u \in \mathcal{N}_{i, q, p}(\Omega)$ if and only if

$$
\begin{aligned}
& \exists\left(\lambda_{n}\right),\left(\mu_{n}\right) \subset \mathscr{M}_{i, q, p}^{0}(\Omega): \\
& \text { (1) } \lambda_{n} \leq \lambda_{n+1} \leq \mu_{n+1} \leq \mu_{n}, \quad n \in \mathbb{N}, \\
& \text { (2) } \bigcap_{n \in \mathbb{N}}\left[\lambda_{n}, \mu_{n}\right]_{\mid V}=\{u\}_{\mid V}, V \subseteq \Omega \text { open, } \\
& \text { (3) }\left[\left\{\left[\lambda_{n}, \mu_{n}\right]: n \in \mathbb{N}\right\}\right] \subseteq \mathscr{F} .
\end{aligned}
$$

Theorem 7. The space $\mathcal{N}_{i, q, p}(\Omega)$ equipped with the uniform convergence structure $\mathscr{J}_{i, q, p}^{\sharp}$ is the uniform convergence space completion of $\mathscr{M}_{i, q, p}^{0}(\Omega)$.

Proof. That $\mathcal{N}_{i, q, p}(\Omega)$ is complete follows immediately by the above remarks. Furthermore, it is clear that the subspace uniform convergence structure on $\mathscr{M L}_{i, q, p}^{0}(\Omega)$ is equal to $\mathscr{J}_{i, q, p}$. To see that $\mathscr{M} \mathscr{L}_{i, q, p}^{0}(\Omega)$ is dense in $\mathcal{N}_{i, q, p}(\Omega)$, consider any $u \in \mathcal{N}_{i, q, p}(\Omega)$. We claim

$$
u=\sup \mathscr{A},
$$

where

$$
\mathscr{A}=\left\{v \in \mathscr{M} \mathscr{L}_{i, q, p}^{0}(\Omega): u \leq v\right\} .
$$

Suppose that this were not the case. Then, since $\mathcal{N} \mathscr{L}(\Omega)$ is Dedekind order complete, it follows that there is some $u_{0} \in$ $\mathscr{N} \mathscr{L}(\Omega)$ so that $u_{0}=\sup \mathscr{A}$. Since $(55)$ does not hold, it follows that $u_{0}$ must satisfy $u>u_{0}$. Furthermore, it is clear from (51) that $u_{0} \in \mathcal{N} \mathscr{L}_{i, q, p}(\Omega)$. In view of (10) and the semicontinuity of $u$ and $u_{0}$ we have

$$
\begin{aligned}
& \exists \epsilon>0: \\
& \forall(y, t) \in W: \\
& \quad u_{0}(y, t)+\epsilon<u(y, t) .
\end{aligned}
$$$$
\exists W \subseteq \Omega \text { nonempty and open : }
$$

From (51) it follows that $W \cap \mathbb{R}^{k-1} \times\left\{t_{0}\right\}=\emptyset$. As such, it follows that $v+(\epsilon / 2) \chi_{W} \in \mathscr{N}_{i, q, p}(\Omega)$ for each $v \in \mathscr{A}$ and that

$$
v+\frac{\epsilon}{2} \chi_{W} \leq u_{0}<u, \quad v \in \mathscr{A} .
$$

It now follows that

$$
v \leq(I \circ S)\left(u_{0}-\frac{\epsilon}{2} \chi_{W}\right)<u_{0}, \quad v \in \mathscr{A},
$$

which is a contradiction. Thus (55) holds. The fact that $\mathscr{M} \mathscr{L}_{i, q, p}^{0}(\Omega)$ is dense in $\mathscr{N}_{i, q, p}(\Omega)$ now follows from the fact that $\mathscr{N} \mathscr{L}(\Omega)$ is order separable [8].

The extension property for uniformly continuous mappings follows by a straightforward argument. 
An important property of the uniform convergence space $\mathscr{M} \mathscr{L}_{i, q, p}^{0}(\Omega)$ and its completion $\mathscr{N}_{i, q, p}(\Omega)$ relates to the inclusion mapping

$$
i: \mathscr{M}_{i, q, p}^{0}(\Omega) \longrightarrow \mathscr{M} \mathscr{L}^{0}(\Omega)
$$

and its extension through uniform continuity

$$
i^{\sharp}: \mathscr{N}_{i, q, p}(\Omega) \longrightarrow \mathscr{N} \mathscr{L}(\Omega) .
$$

Indeed, it is clear form Definitions 1 and 3 that the mapping (60) is in fact uniformly continuous. Similarly, the inclusion mapping

$$
i_{0}: \mathcal{N} \mathscr{L}_{i, q, p}(\Omega) \longrightarrow \mathscr{N} \mathscr{L}(\Omega)
$$

is uniformly continuous. Since the mappings (61) and (62) coincide on a dense subset of $\mathcal{N} \mathscr{L}_{i, q, p}(\Omega)$, it follows that (61) is simply the inclusion mapping (62). This is related to the issue of consistency of generalized solutions of (29) to (30), which we construct in the sequel, with solutions in the space $\mathcal{N}_{\mathscr{L}^{m}}(\Omega)^{K}$; see [10]. We will discuss this in some detail in what follows, after the uniform convergence structure on $\mathscr{M} \mathscr{L}_{\mathbf{g}}^{m}(\Omega)$ has been introduced.

In this regard, the uniform convergence structure $\mathscr{J}_{\mathbf{g}}$ on $\mathscr{M}_{\mathscr{L}_{\mathbf{g}}^{m}}(\Omega)$ is defined as the initial uniform convergence structure with respect to the mappings (35) to (37). That is, a filter $\mathscr{U}$ on $\mathscr{M}_{\mathscr{L}_{\mathbf{g}}^{m}}(\Omega) \times \mathscr{M}_{\mathscr{L}_{\mathbf{g}}^{m}}(\Omega)$ belongs to $\mathscr{J}_{\mathbf{g}}$ if and only if

$$
\forall i=1, \ldots, K:\left(\mathscr{D}_{i, t}^{m} \times \mathscr{D}_{i, t}^{m}\right)(\mathcal{U}) \in \mathscr{J}_{o}
$$

and

$$
\begin{aligned}
& \forall 0 \leq p<m: \\
& \forall q \in \mathbb{N}^{k-1}, \quad 0<|q|+p \leq m: \\
& \forall i=1, \ldots, K: \\
& \quad\left(\mathscr{D}_{i, y t}^{q p} \times \mathscr{D}_{i, y t}^{q p}\right)(\mathcal{U} \times \mathcal{U}) \in \mathcal{J}_{i, q, p} .
\end{aligned}
$$

Clearly the family consisting of the mappings (35) through (37) separates the points of $\mathscr{M}_{\mathscr{L}_{\mathbf{g}}^{m}}(\Omega)$. As such, the uniform convergence structure $\mathscr{J}_{\mathrm{g}}$ is uniformly Hausdorff. In particular, see [14], the mapping

$$
\mathbf{D}: \mathscr{M}_{\mathscr{L}_{\mathrm{g}}^{m}}(\Omega) \longrightarrow\left(\prod \mathscr{M}_{i, q, p}^{0}(\Omega)\right) \times \mathscr{M} \mathscr{L}^{0}(\Omega)^{K}
$$

which is defined through

$$
\mathbf{D}(\mathbf{u})=\left(\ldots, \mathscr{D}_{i, y t}^{q p} \mathbf{u}, \ldots, \mathscr{D}_{i, t}^{m} \mathbf{u}, \ldots\right)
$$

is a uniformly continuous embedding. As such, it follows that the mapping (66) extends to an injective, uniformly continuous mapping

$$
\mathbf{D}^{\sharp}: \mathcal{N} \mathscr{L}_{\mathbf{g}}^{m}(\Omega) \longrightarrow\left(\prod \mathcal{N} \mathscr{L}_{i, q, p}(\Omega)\right) \times \mathcal{N} \mathscr{L}(\Omega)^{K},
$$

where $\mathcal{N}_{\mathbf{g}}^{m}(\Omega)$ denotes the uniform convergence space completion of $\mathscr{M}_{\mathscr{L}_{\mathbf{g}}^{m}}(\Omega)$. In particular, for each $i=1, \ldots, K$, every $0 \leq p<m$, and each $q \in \mathbb{N}^{k-1}$ such that $0 \leq p+|q| \leq m$ the diagrams

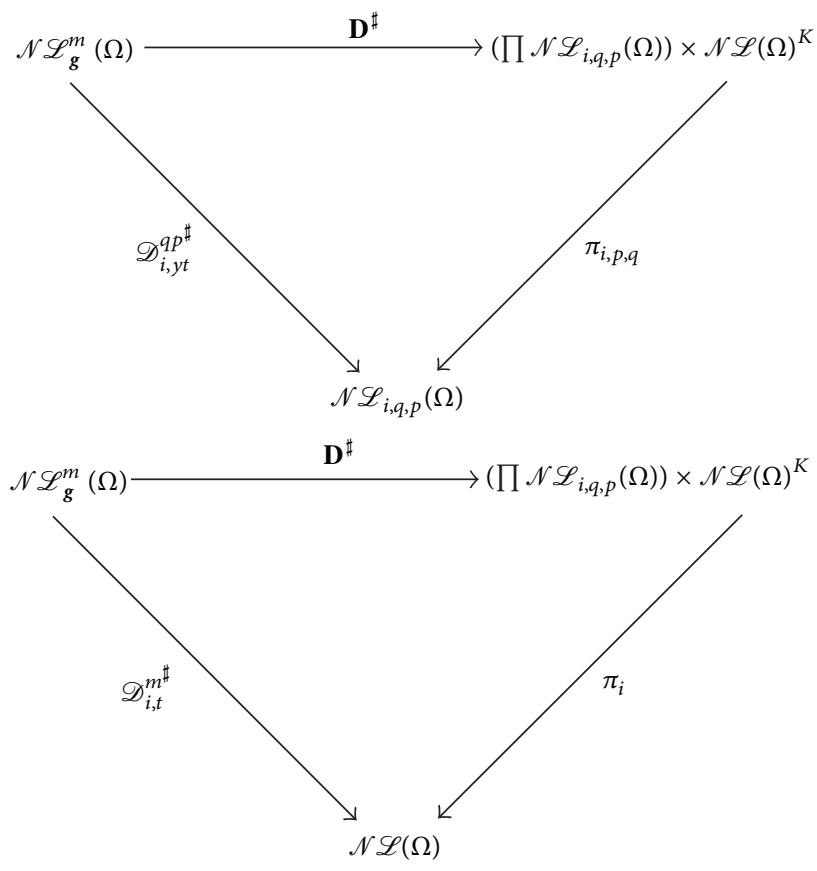

commute, with $\pi_{i, q, p}$ and $\pi_{i}$ the appropriate projections and $\mathscr{D}_{i, y t}^{q p \sharp}$ and $\mathscr{D}_{i, t}^{m \sharp}$ the extensions through uniform continuity of the mappings (35) and (37), respectively.

The meaning of the diagram (68) is twofold. Firstly, it explains the regularity of generalized functions in $\mathcal{N}_{\mathbf{g}}^{m}(\Omega)$. In particular, each generalized partial derivative of a generalized function $\mathbf{u}^{\sharp} \in \mathcal{N}_{\mathbf{g}}^{m}(\Omega)$ is a nearly finite normal lower semicontinuous function. Therefore, each such generalized function may be represented as an element of the space $\left(\prod \mathcal{N} \mathscr{L}_{i, p}(\Omega)\right) \times \mathscr{N} \mathscr{L}(\Omega)^{K}$ in a canonical way. Secondly, these diagrams state that each generalized function $\mathbf{u}^{\sharp} \in \mathcal{N}_{\mathscr{L}_{\mathbf{g}}^{m}}(\Omega)$ satisfies the initial condition (30) in the sense that

$$
\begin{aligned}
& \forall i=1, \ldots, K: \\
& \forall 0 \leq p<m: \\
& \forall q \in \mathbb{N}^{k-1}, \quad 0 \leq p+|q| \leq m: \\
& \quad \mathscr{D}_{i, y t}^{q p \sharp} \mathbf{u}^{\sharp}\left(t_{0}, y\right)=D^{q} g_{p, i}\left(t_{0}, y\right), \quad y \in \mathbb{R}^{k-1} .
\end{aligned}
$$

\section{Existence of Generalized Solutions}

With the system of nonlinear PDEs (29) we may associate a mapping

$$
\mathbf{T}: \mathscr{M}_{\mathscr{L}_{\mathbf{g}}^{m}}(\Omega) \longrightarrow \mathscr{M} \mathscr{L}^{0}(\Omega)^{K}
$$


the components of which are defined through

$$
\begin{aligned}
T_{j}: \mathscr{M}_{\mathbf{g}}^{m}(\Omega) & \ni \mathbf{u} \\
& \longmapsto(I \circ S)\left(\mathscr{D}_{j, t}^{m} \mathbf{u}+G_{j}\left(\cdot, \cdot, \ldots, \mathscr{D}_{i, y t}^{p q} \mathbf{u}, \ldots\right)\right) \\
& \in \mathscr{M L}^{0}(\Omega) .
\end{aligned}
$$

We arrive at the notion of generalized solution of the initial value problem (29) and (30) in the context of the space $\mathcal{N} \mathscr{L}_{\mathrm{g}}^{m}(\Omega)$ by suitably extending the mapping (70) to a mapping

$$
\mathbf{T}^{\sharp}: \mathscr{N}_{\mathbf{g}}^{m}(\Omega) \longrightarrow \mathscr{N} \mathscr{L}(\Omega)^{K}
$$

Such an extension is obtained through the uniform continuity of the mapping (70). In this regard, we have the following.

Theorem 8. The mapping (70) is uniformly continuous.

Proof. It follows from (60) through (61) that the inclusion mapping

$$
i: \mathscr{M}_{\mathbf{g}}^{m}(\Omega) \longrightarrow \mathscr{M}^{m} \mathscr{L}^{m}(\Omega)^{K}
$$

is uniformly continuous. The result now follows from the commutative diagram

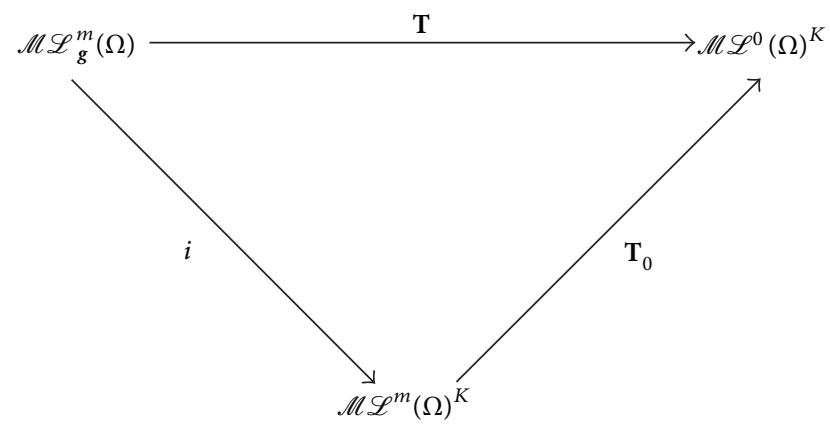

and the uniform continuity of $\mathbf{T}_{0}$, which is the mapping defined on $\mathscr{M}_{\mathscr{L}^{m}}(\Omega)^{K}$ through the nonlinear partial differential operator; see [10].

In view of Theorem 8 the mapping (70) extends in a unique way to a uniformly continuous mapping (72). As such, the generalized initial value problem corresponding to (29) and (30) is given by the single equation

$$
\mathbf{T}^{\sharp} \mathbf{u}^{\sharp}=\mathbf{0},
$$

where $\mathbf{0}$ denotes the element in $\mathscr{N} \mathscr{L}(\Omega)^{K}$ with all components identically 0 . A solution of (75) is interpreted as a generalized solution of (29) through (30) based on the fact that each solution of (75) satisfies the initial condition in a suitable generalized sense, as mentioned in (69). Furthermore, in view of (60) to (62) and the diagram (74) we obtain the commutative diagram

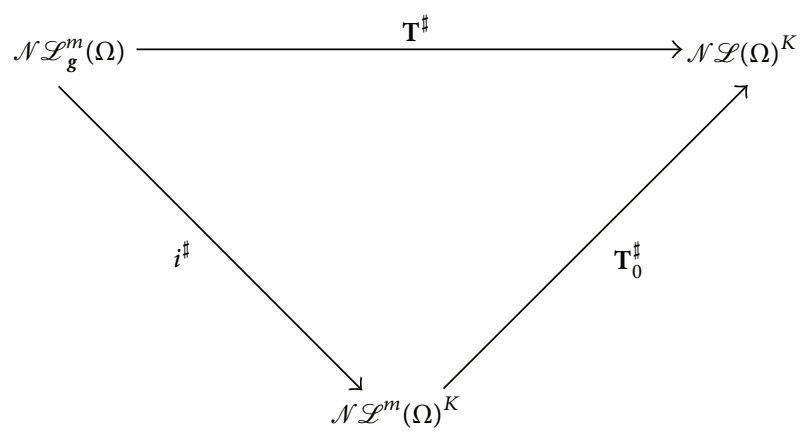

with $i^{\sharp}$ injective and $\mathbf{T}_{0}^{\sharp}$ the uniformly continuous extension of the mapping

$$
\mathrm{T}_{0}: \mathscr{M} \mathscr{L}^{m}(\Omega)^{K} \longrightarrow \mathscr{M} \mathscr{L}^{0}(\Omega)^{K}
$$

associated with the system of nonlinear PDEs (29). In particular, the mapping $i^{\sharp}$ is the inclusion mapping. As such, each solution $\mathbf{u}^{\sharp} \in \mathscr{N}_{\mathbf{g}}^{m}(\Omega)$ of (75) is a generalized solution of the system of nonlinear PDEs (29) in the sense of the spaces of generalized functions introduced in [10]. The main result of this section is the following.

Theorem 9. For each $0 \leq p<m$, let $\mathbf{g}_{p} \in \mathscr{C}^{m-p}\left(\mathbb{R}^{k-1}\right)^{K}$. Then there is some $\mathbf{u}^{\sharp} \in \mathcal{N}_{\mathscr{L}_{\mathbf{g}}^{m}}(\Omega)$ so that

$$
\mathbf{T}^{\sharp} \mathbf{u}^{\sharp}=\mathbf{0} .
$$

Proof. Let us express $\Omega=\mathbb{R}^{k-1} \times \mathbb{R}$ as

$$
\Omega=\bigcup_{v \in \mathbb{N}} C_{v}
$$

where, for $v \in \mathbb{N}$, the compact set $C_{v}$ is an $n$-dimensional interval

$$
C_{v}=\left[a_{v}, b_{v}\right]
$$

with $a_{v}=\left(a_{v, 1}, \ldots, a_{v, n}\right), b_{v}=\left(b_{v, 1}, \ldots, b_{v, n}\right) \in \mathbb{R}^{k}$, and $a_{v, j} \leq$ $b_{v, j}$ for every $j=1, \ldots, n$. We assume that $\left\{C_{v}: v \in \mathbb{N}\right\}$ is locally finite, that is:

$$
\begin{aligned}
& \forall x \in \Omega: \\
& \exists V \subseteq \Omega \text { a neighborhood of } x: \\
& \quad\left\{v \in \mathbb{N}: C_{v} \cap V \neq \emptyset\right\} \text { is finite. }
\end{aligned}
$$

Such a partition of $\Omega$ exists; see, for instance [16]. We also assume that, for each $v \in \mathbb{N}$,

$$
\mathcal{S} \cap C_{v}=\emptyset
$$

or

$$
\mathcal{S} \cap \operatorname{IntC}_{v} \neq \emptyset,
$$


where $\mathcal{S}$ is the noncharacteristic hypersurface $\mathcal{S}=\left\{\left(y, t_{0}\right)\right.$ : $\left.y \in \mathbb{R}^{k-1}\right\}$. For the sake of convenience, let us write $x=(y, t)$ for each $(y, t) \in \mathbb{R}^{k-1} \times \mathbb{R}$. Let $\mathbf{F}: \Omega \times \mathbb{R}^{M} \rightarrow \mathbb{R}^{K}$ be the mapping that defines the nonlinear operator $\mathbf{T}$ through $\mathbf{T}(x, D) \mathbf{u}(x)=\mathbf{F}\left(x, \ldots, D^{\alpha} u_{i}(x), \ldots\right)$. Fix $v \in \mathbb{N}$ such that (83) is satisfied. In view of the fact that the mapping $\mathbf{F}$ is both open and surjective, we have

$$
\begin{aligned}
& \forall x_{1}=\left(y_{1}, t_{1}\right) \in C_{v}: \\
& \exists \xi\left(x_{1}\right) \in \mathbb{R}^{M}, \quad \mathbf{F}\left(x_{1}, \xi\left(x_{1}\right)\right)=0: \\
& \exists \delta, \epsilon>0:
\end{aligned}
$$$$
\text { (1) }\left\{(x, 0):\left\|x-x_{1}\right\|<\delta\right\}
$$$$
c \operatorname{int}\left\{\begin{array}{l|l}
(x, \mathbf{F}(x, \xi)) & \begin{array}{c}
\left\|x-x_{1}\right\|<\delta \\
\left\|\xi-\xi\left(x_{1}\right)\right\|<\epsilon
\end{array}
\end{array}\right\},
$$

(2) $\mathbf{F}: B_{\delta}\left(x_{1}\right) \times B_{2 \epsilon}\left(\xi\left(x_{1}\right)\right) \longrightarrow \mathbb{R}^{K}$ open.

In particular, if $t_{1}=t_{0}$, we may take $\xi\left(x_{1}\right)=\left(\xi_{i}^{q, p}, \xi_{i}^{m}\right)$ such that

$$
\begin{aligned}
& \forall i=1, \ldots, K: \\
& \forall 0 \leq p<m: \\
& \forall q \in \mathbb{N}^{k-1}, \quad 0<|q|+p \leq m: \\
& \xi_{i}^{q, p}=D^{q} g_{p, i}\left(y_{1}\right) .
\end{aligned}
$$

For each $x_{1} \in C_{v}$, fix $\xi\left(x_{1}\right) \in \mathbb{R}^{M}$ in (84) so that (85) is satisfied in case $t_{1}=t_{0}$. Since $C_{v}$ is compact, it follows from (84) that

$$
\begin{aligned}
& \exists \delta>0: \\
& \forall x_{1} \in C_{v}: \\
& \exists \epsilon_{x_{1}}>0:
\end{aligned}
$$$$
\text { (1) }\left\{(x, 0):\left\|x-x_{1}\right\|<\delta\right\}
$$$$
c \operatorname{int}\left\{\begin{array}{l|l}
(x, \mathbf{F}(x, \xi)) & \begin{array}{c}
\left\|x-x_{1}\right\|<\delta \\
\left\|\xi-\xi\left(x_{1}\right)\right\|<\epsilon_{x_{1}}
\end{array}
\end{array}\right\},
$$

(2) $\mathbf{F}: B_{\delta}\left(x_{1}\right) \times B_{2 \epsilon_{x_{0}}}\left(\xi\left(x_{1}\right)\right) \longrightarrow \mathbb{R}^{K}$ open.

Subdivide $C_{v}$ into $n$-dimensional intervals $I_{v, 1}, \ldots, I_{v, \mu_{v}}$ with diameter not exceeding $\delta$ such that their interiors are pairwise disjoint and, for each $j=1, \ldots, \mu_{v}$,

$$
I_{v, j} \cap \mathcal{S}=\emptyset,
$$

or

$$
\text { int } I_{v, j} \cap \mathcal{S} \neq \emptyset \text {. }
$$

If $a_{v, j}$ with $j=1, \ldots, \mu_{\nu}$ is the center of the interval $I_{v, j}$ that satisfies (87), then by (86) we have

$$
\begin{aligned}
& \exists \epsilon_{v, j}>0: \\
& \text { (1) }\left\{(x, 0): x \in I_{v, j}\right\} \\
& \quad \quad \operatorname{int}\left\{(x, \mathbf{F}(x, \xi)) \mid \begin{array}{l}
x \in I_{v, j} \\
\left\|\xi-\xi\left(a_{v, j}\right)\right\|<\epsilon_{v, j}
\end{array}\right\}, \\
& \text { (2) } \mathbf{F}: I_{v, j} \times B_{2 \epsilon_{v, j}}\left(\xi\left(a_{v, j}\right)\right) \longrightarrow \mathbb{R}^{K} \text { open. }
\end{aligned}
$$

On the other hand, if $I_{v, j}$ satisfies (88), set $a_{v, j}$ equal to the midpoint of $\mathcal{S} \cap I_{v, j}$. Then we obtain (89) by (86) such that (85) also holds. Take $0<\gamma<1$ arbitrary but fixed. In view of [9, Lemma 5] and (89), we have

$$
\begin{aligned}
& \forall x_{1} \in I_{v, j}: \\
& \exists \mathbf{U}_{x_{1}}=\mathbf{U} \in \mathscr{C}^{m}\left(\mathbb{R}^{k}\right)^{K}: \\
& \exists \delta=\delta_{x_{1}}>0: \\
& x \in B_{\delta}\left(x_{1}\right) \cap I_{\nu, j} \\
& \quad \Longrightarrow\left(\begin{array}{c}
(1)\left(D^{\alpha} U_{i}(x)\right)_{i \leq K}^{|\alpha| \leq m} \in B_{\epsilon_{v, j}}\left(\xi\left(a_{\nu, j}\right)\right) \\
(2) i \leq K \Longrightarrow \gamma<T_{i}(x, D) \mathbf{U}(x)<0
\end{array}\right),
\end{aligned}
$$

with $\alpha=(q, p)$. Furthermore, if $I_{v, j}$ satisfies (88), then we also have

$$
\begin{aligned}
& \forall i=1, \ldots, K: \\
& \forall 0 \leq p<m: \\
& \forall q \in \mathbb{N}^{k-1}, \quad 0<|q|+p \leq m: \\
& \forall y \in \mathbb{R}^{k-1}: \\
& D_{y t}^{p q} U_{i}\left(y, t_{0}\right)=D^{q} g_{p, i}(y) .
\end{aligned}
$$

Indeed, in this case we may simply set

$$
U_{i}(y, t)=\sum_{p=0}^{m-1}\left(t-t_{0}\right)^{p} g_{p, i}(y)+w_{i}(t)
$$

for a suitable function $w_{i} \in \mathscr{C}^{m}(\mathbb{R})$ that satisfies

$$
\forall 0 \leq p<m: w_{i}^{(p)}\left(t_{0}\right)=0 .
$$

As above, we may subdivide $I_{v, j}$ into pairwise disjoint, $n$ dimensional intervals $J_{v, j, 1}, \ldots, J_{v, j, \mu_{v, j}}$ so that for $k=$ $1, \ldots, \mu_{v, j}$ we have

$$
\begin{aligned}
& \exists \mathbf{U}^{v, j, k}=\mathbf{U} \in \mathscr{C}^{m}\left(\mathbb{R}^{k}\right)^{K}: \\
& \forall x \in J_{v, j, k}: \\
& \text { (1) }\left(D^{\alpha} U_{i}(x)_{i \leq K}^{|\alpha| \leq m}\right) \in B_{\epsilon_{v, j}}\left(\xi\left(a_{v, j}\right)\right), \quad|\alpha| \leq m, \\
& \text { (2) } i \leq K \Longrightarrow f_{i}(x)-\gamma<T_{i}(x, D) \mathbf{U}(x)<f_{i}(x),
\end{aligned}
$$


and

$$
J_{v, j, k} \cap \mathcal{S}=\emptyset,
$$

or

$$
\text { int } I_{v, j, k} \cap \mathcal{S} \neq \emptyset \text {. }
$$

Furthermore, whenever $J_{v, j, k}$ satisfies (96), we have

$$
\begin{aligned}
& \forall i=1, \ldots, K: \\
& \forall 0 \leq p<m: \\
& \forall q \in \mathbb{N}^{k-1}, \quad 0<|q|+p \leq m: \\
& \forall y \in \mathbb{R}^{k-1}: \\
& \quad D_{y t}^{q p} U_{i}\left(y, t_{0}\right)=D^{q} g_{p, i}(y) .
\end{aligned}
$$

Set

$$
\begin{gathered}
\Gamma_{1}=\Omega \backslash\left(\bigcup_{v \in \mathbb{N}}\left(\bigcup_{j=1}^{\mu_{v}}\left(\bigcup_{k=1}^{\mu_{v, j}} \operatorname{int} J_{v, j, k}\right)\right),\right. \\
\mathbf{V}_{1}=\sum_{v \in \mathbb{N}}\left(\sum_{j=1}^{\mu_{v}}\left(\sum_{k=1}^{\mu_{v, j}} \chi_{J_{v, j, k}} \mathbf{U}_{v, j, k}\right)\right),
\end{gathered}
$$

where $\chi_{J_{v, j, k}}$ is the characteristic function of $J_{v, j, k}$. Then $\Gamma_{1}$ is closed nowhere dense, and $\mathbf{V}_{1} \in \mathscr{C}^{m}\left(\Omega \backslash \Gamma_{1}\right)^{K}$. Furthermore, $\mathcal{S} \cap \Gamma_{1}$ is closed nowhere dense in $\mathcal{S}$ and

$$
\begin{aligned}
& \forall i=1, \ldots, K: \\
& \forall 0 \leq p<m: \\
& \forall q \in \mathbb{N}^{k-1}, \quad 0<|q|+p \leq m: \\
& \forall\left(y, t_{0}\right) \in \mathcal{S} \backslash\left(\mathcal{S} \cap \Gamma_{1}\right): \\
& \quad D_{y t}^{q p} V_{1, i}\left(y, t_{0}\right)=D^{q} g_{p, i}(y) .
\end{aligned}
$$

In view of (94) we have, for each $i=1, \ldots, K$

$$
-\gamma<T_{i}(x, D) \mathbf{V}_{1}(x)<0, \quad x \in \Omega \backslash \Gamma_{1} .
$$

Furthermore, for each $v \in \mathbb{N}$, for each $j=1, \ldots, \mu_{v}$, each $k=1, \ldots, \mu_{v, j}$, each $|\alpha| \leq m$, and every $i=1, \ldots, K$ we have

$$
x \in \text { int } J_{v, j, k} \Longrightarrow \xi_{i}^{\alpha}\left(a_{v, j}\right)-\epsilon<D^{\alpha} V_{1, i}(x)<\xi_{i}^{\alpha}\left(a_{v, j}\right)+\epsilon .
$$

For $0 \leq p<m$, define the functions $\lambda_{1, i}^{\alpha}, \mu_{1, i}^{\alpha} \in \mathscr{C}^{0}\left(\Omega \backslash \Gamma_{1}\right)$, where $\alpha=(p, q)$ with $|q|=0$, as

$$
\begin{gathered}
\lambda_{1, i}^{\alpha}(x)= \begin{cases}\xi_{i}^{\alpha}\left(a_{v, j}\right)-2 \epsilon_{v, j} & \text { if } x \in \operatorname{int} I_{v, j, k}, \\
D_{t}^{p} V_{1, i}(y, t)-v_{v, j}(t) & I_{v, j, k} \cap \mathcal{S}=\emptyset \\
& \text { if } x \in \operatorname{int} I_{v, j, k}, \\
\mu_{v, j, k} \cap \mathcal{S} \neq \emptyset,\end{cases} \\
\mu_{1, i}^{\alpha}(x)= \begin{cases}\xi_{i}^{\alpha}\left(a_{v, j}\right)+2 \epsilon_{v, j} & \text { if } x \in \operatorname{int} I_{v, j, k}, \\
D_{t}^{p} V_{1, i}(y, t)+v_{v, j}(t) & I_{v, j, k} \cap \mathcal{S}=\emptyset \\
& \text { if } x \in \operatorname{int} I_{v, j, k},\end{cases} \\
I_{v, j, k} \cap \mathcal{S} \neq \emptyset .
\end{gathered}
$$

Here $v_{v, j}$ is a continuous, real valued function on $\mathbb{R}$ such that

$$
\begin{gathered}
v_{v, j}\left(t_{0}\right)=0, \\
0<v_{v, j}(t)<2 \epsilon_{v, j}, \quad t \neq 0 .
\end{gathered}
$$

For all other $\alpha$, consider the functions

$$
\begin{array}{ll}
\lambda_{1, i}^{\alpha}(x)=\xi_{i}^{\alpha}\left(a_{v, j}\right)-2 \epsilon_{v, j} & \text { if } x \in \operatorname{int} I_{v, j}, \\
\mu_{1, i}^{\alpha}(x)=\xi_{i}^{\alpha}\left(a_{v, j}\right)+2 \epsilon_{v, j} & \text { if } x \in \operatorname{int} I_{v, j} .
\end{array}
$$

Then it follows by (101) that

$$
\begin{gathered}
\lambda_{1, i}^{\alpha}(x)<D^{\alpha} V_{1, i}(x)<\mu_{1, i}^{\alpha}(x), \quad x \in \Omega \backslash \Gamma_{1}, \\
\mu_{1, i}^{\alpha}(x)-\lambda_{1, i}^{\alpha}(x)<4 \epsilon_{v, j}, \quad x \in \operatorname{int} I_{v, j} .
\end{gathered}
$$

Applying (89) restricted to $\Omega \backslash \Gamma_{1}$, and proceeding in a fashion similar as above, we may construct, for each $n \in \mathbb{N}$ such that $n>1$, a closed nowhere dense set $\Gamma_{n} \subset \Omega$ such that

$$
\Gamma_{n} \cap \mathcal{S} \text { closed nowhere dense in } \mathcal{S} \text {, }
$$

a function $\mathbf{V}_{n} \in \mathscr{C}^{m}\left(\Omega \backslash \Gamma_{n}\right)^{K}$ and functions $\lambda_{n, i}^{\alpha}, \mu_{n, i}^{\alpha} \in \mathscr{C}^{0}(\Omega \backslash$ $\Gamma_{n}$ ) so that, for each $i=1, \ldots, K$,

$$
-\frac{\gamma}{n}<T_{i}(x, D) \mathbf{V}_{n}(x)<0, \quad x \in \Omega \backslash \Gamma_{n}
$$

and for every $|\alpha| \leq m$

$$
\begin{aligned}
\lambda_{n-1, i}^{\alpha}(x) & <\lambda_{n, i}^{\alpha}(x)<D^{\alpha} V_{n, i}(x)<\mu_{n, i}^{\alpha}(x) \\
& <\mu_{n-1, i}^{\alpha}(x), \quad x \in \Omega \backslash \Gamma_{n}, \\
\mu_{n, i}^{\alpha}(x)-\lambda_{n, i}^{\alpha}(x)< & \frac{4 \epsilon_{v, j}}{n}, \quad x \in\left(\operatorname{int} I_{v, j}\right) \cap\left(\Omega \backslash \Gamma_{n}\right) .
\end{aligned}
$$

Furthermore, for each $0 \leq p<m$ and $q \in \mathbb{N}^{k-1}$ so that $0 \leq$ $|q|+p \leq m$ we have

$$
\begin{aligned}
D_{y t}^{q p} V_{n, i}\left(y, t_{0}\right) & =\lambda_{n, i}^{\alpha}\left(y, t_{0}\right)=\mu_{n, i}^{\alpha}\left(y, t_{0}\right) \\
& =D^{q} g_{p, i}(y), \quad\left(y, t_{0}\right) \notin \mathcal{S} \cap \Gamma_{n},
\end{aligned}
$$

where $\alpha=(p, q)$.

Notice that the functions $\mathbf{u}_{n}$, the components of which are defined through

$$
u_{n, i}=(I \circ S)\left(V_{n, i}\right),
$$

belong to $\mathscr{M}_{\mathscr{L}_{\mathbf{g}}^{m}}(\Omega)$. In view of (108) it follows that the functions $\bar{\lambda}_{n, i}^{\alpha}, \bar{\mu}_{n, i}^{\alpha} \in \mathscr{M} \mathscr{L}^{0}(\Omega)$, which are defined as

$$
\bar{\lambda}_{n, i}^{\alpha}=(I \circ S)\left(\lambda_{n, i}^{\alpha}\right), \quad \bar{\mu}_{n, i}^{\alpha}=(I \circ S)\left(\mu_{n, i}^{\alpha}\right),
$$

satisfy

$$
\bar{\lambda}_{n-1, i}^{\alpha} \leq \bar{\lambda}_{n, i}^{\alpha} \leq \mathscr{D}^{\alpha} u_{n, i} \leq \bar{\mu}_{n, i}^{\alpha} \leq \bar{\mu}_{n-1, i}^{\alpha} .
$$


Furthermore, in case $\alpha=(p, q)$ with $0 \leq p<m$ and $q \in \mathbb{N}^{k-1}$ such that $0 \leq p+|q| \leq m$, then $\bar{\lambda}_{n, i}^{\alpha}, \bar{\mu}_{n, i}^{\alpha} \in$ $\mathscr{M} \mathscr{L}_{i, q, p}^{0}(\Omega)$. It now follows from (109) that the sequence $\left(\mathbf{u}_{n}\right)$ is a Cauchy sequence in $\mathscr{M}_{\mathbf{L}}^{m}(\Omega)$. Moreover, (107) implies that the sequence $\left(\mathbf{T u}_{n}\right)$ converges to $\mathbf{0}$ in $\mathscr{M} \mathscr{L}^{0}(\Omega)^{K}$. The result now follows from Theorem 8 .

We have shown that the initial value problem (29) through (30) admits a generalized solution in the space $\mathscr{N} \mathscr{L}_{\mathbf{g}}^{m}(\Omega)$. In particular, and in view of the commutative diagram (74), the generalized solution constructed in Theorem 9 is a generalized solution of the system of nonlinear PDEs in the sense of the spaces of generalized functions introduced in [10]. Furthermore, this solution satisfies the initial condition (30) in the sense that

$$
\begin{aligned}
& \forall 0 \leq p<m: \\
& \forall q \in \mathbb{N}^{k-1}, \quad 0 \leq|q|+p \leq m: \\
& \forall y \in \mathbb{R}^{k-1}: \\
& \mathscr{D}_{y t, i}^{q p \sharp} \mathbf{u}^{\sharp}\left(y, t_{0}\right)=D^{q} g_{p, i}(y) .
\end{aligned}
$$

Furthermore, it follows by (9) that the singularity set

$$
\left\{(y, t) \in \Omega \mid \begin{array}{l}
\exists|\alpha| \leq m: \\
\exists i=1, \ldots, K: \\
\mathscr{D}_{i}^{\alpha \sharp} \mathbf{u} \text { not continuous at }(y, t)
\end{array}\right\}
$$

of the solution $\mathbf{u}^{\sharp}$ is of first Baire category. In particular, there exists a residual subset $R$ of $\Omega$ such that each generalized partial derivative of $\mathbf{u}^{\sharp}$ is continuous at every point of $R$.

As mentioned in Section 1, it is known [3] that if the mapping G : $\Omega \times \mathbb{R}^{M} \rightarrow \mathbb{R}^{K}$ in (29), as well as the initial data (30) is real analytic, then the initial value problem admits an analytic solution on a neighborhood of the noncharacteristic hypersurface $\mathcal{S}$. Furthermore, Rosinger [4] showed that such an initial value problem admits a generalized solution $\mathbf{u}$, in a suitable differential algebra of generalized functions, which is analytic everywhere except on a closed nowhere dense set. Since this solution is analytic in a neighborhood of $\mathcal{S}$, it follows that $\mathbf{u} \in \mathscr{M}_{\mathscr{L}_{\mathbf{g}}^{m}}^{m}(\Omega)$ and satisfies

$$
\mathbf{T u}=\mathbf{0} .
$$

As such, $\mathbf{u}$ is also a solution in the sense discussed in this paper.

It should be noted that the customary spaces of generalized functions that are typical in the study of nonlinear PDEs may fail to contain generalized solutions of the initial value problem (29) to (30). Indeed, it has been shown that some of these spaces, such as spaces of distributions, fail to contain generalized solutions even of $\mathscr{C}^{\infty}$-smooth linear PDEs; see, for instance [17]. Theorem 9 is therefore a first in the literature. Namely, it is the first extension of the CauchyKovalevskaia theorem, within a suitable space of generalized functions, on its own general and type independent grounds, to equations that are not analytic.
The extent to which the solution constructed here may be interpreted as a classical solution on some part of the domain of definition of the system of equations is unknown at present. Furthermore, it is unknown whether or not, in general, the solution may be interpreted in terms of any of the spaces of generalized functions that are typical in the study of linear and nonlinear PDEs.

\section{Conclusion}

In this paper we have shown how the methods developed in [10] may be modified in order to incorporate also initial and/or boundary value problems. In this regard, generalized solutions of a large class of nonlinear initial value problems are constructed. It should be noted that the techniques used to obtain the existence of solution are essentially the same as those used in [10] for the free problem. In this way, we come to appreciate another advantage of solving nonlinear PDEs in the spaces of generalized functions used here and in [10]. Namely, and in contradistinction with the usual functional analytic methods, initial value problems do not result in significant additional complications in the solution method.

\section{References}

[1] V. I. Arnold, Lectures on Partial Differential Equations, Springer, Berlin, Germany, 2004.

[2] L. C. Evans, Partial Differential Equations, vol. 19 of Graduate Studies in Mathematics, American Mathematical Society, Providence, RI, USA, 1998.

[3] S. Kovalevskaia, "Zur Theorie der partiellen differentialgleichung," Journal für die reine und angewandte Mathematik, vol. 80, pp. 1-32, 1875.

[4] E. E. Rosinger, "Global version of the Cauchy-Kovalevskaia theorem for nonlinear PDEs," Acta Applicandae Mathematicae, vol. 21, no. 3, pp. 331-343, 1990.

[5] J.-F. Colombeau, New Generalized Functions and Multiplication of Distributions, vol. 84, North-Holland, Amsterdam, The Netherlands, 1984.

[6] M. B. Oberguggenberger and E. E. Rosinger, Solution of Continuous Nonlinear PDEs through Order Completion, vol. 181, NorthHolland, Amsterdam, The Netherlands, 1994.

[7] R. Anguelov and E. E. Rosinger, "Solving large classes of nonlinear systems of PDEs," Computers \& Mathematics with Applications, vol. 53, no. 3-4, pp. 491-507, 2007.

[8] J. H. van der Walt, "The uniform order convergence structure on $M L(X)$," Quaestiones Mathematicae, vol. 31, no. 1, pp. 55-77, 2008.

[9] J. H. van der Walt, "The order completion method for systems of nonlinear PDEs: pseudo-topological perspectives," Acta Applicandae Mathematicae, vol. 103, no. 1, pp. 1-17, 2008.

[10] J. H. van der Walt, "The order completion method for systems of nonlinear PDEs revisited," Acta Applicandae Mathematicae, vol. 106, no. 2, pp. 149-176, 2009.

[11] R. Baire, Lecons Sur Les Fonctions Discontinues, Collection Borel, Paris, France, 1905.

[12] R. Anguelov, "Dedekind order completion of $C(X)$ by Hausdorff continuous functions," Quaestiones Mathematicae, vol. 27, no. 2, pp. 153-169, 2004. 
[13] R. P. Dilworth, "The normal completion of the lattice of continuous functions," Transactions of the American Mathematical Society, vol. 68, pp. 427-438, 1950.

[14] J. H. van der Walt, "The completion of uniform convergence spaces and an application to nonlinear PDEs," Quaestiones Mathematicae, vol. 32, no. 3, pp. 371-395, 2009.

[15] R. Beattie and H.-P. Butzmann, Convergence Structures and Applications to Functional Analysis, Kluwer Academic Publishers, Dordrecht, The Netherlands, 2002.

[16] O. Forster, Analysis 3, Integralrechnung im $\mathbb{R}^{n}$ mit Anwendungen, vol. 52, Vieweg, Braunschweig, Germany, 1981.

[17] L. Hörmander, Linear Partial Differential Operators, Springer, Berlin, Germany, 1976. 


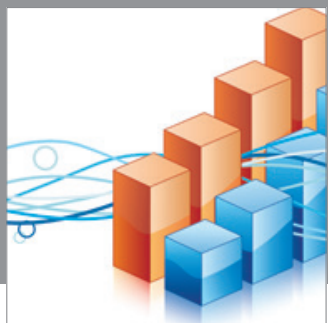

Advances in

Operations Research

mansans

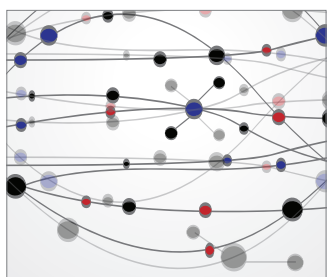

The Scientific World Journal
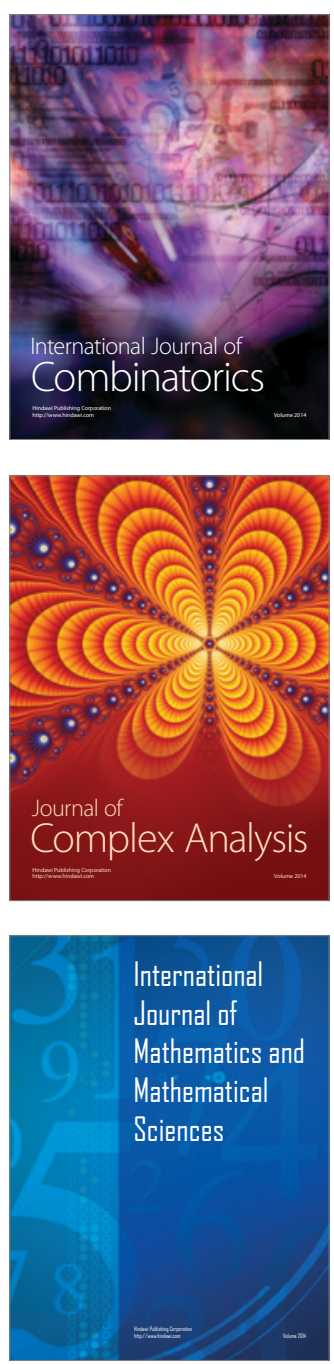
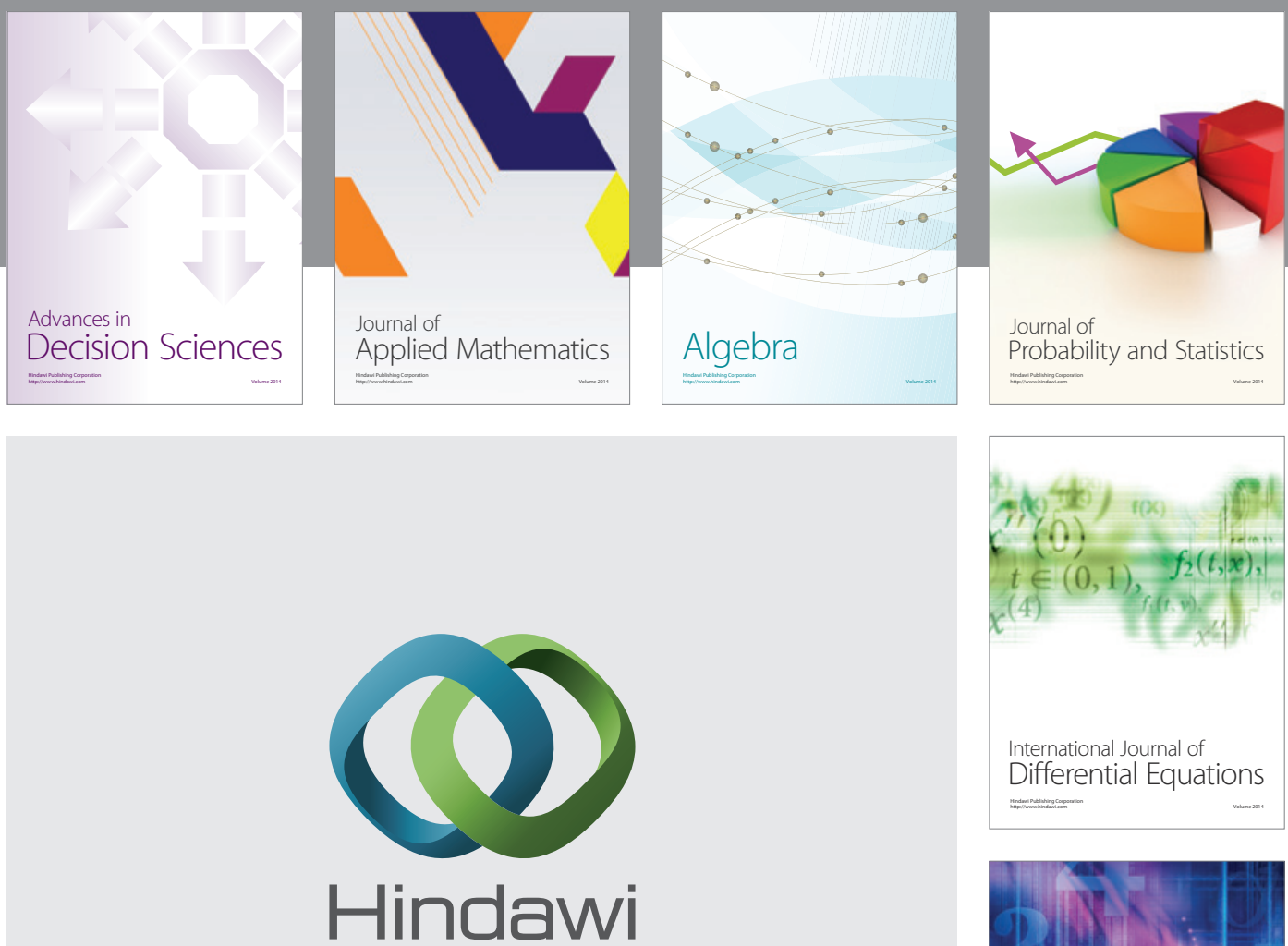

Submit your manuscripts at http://www.hindawi.com
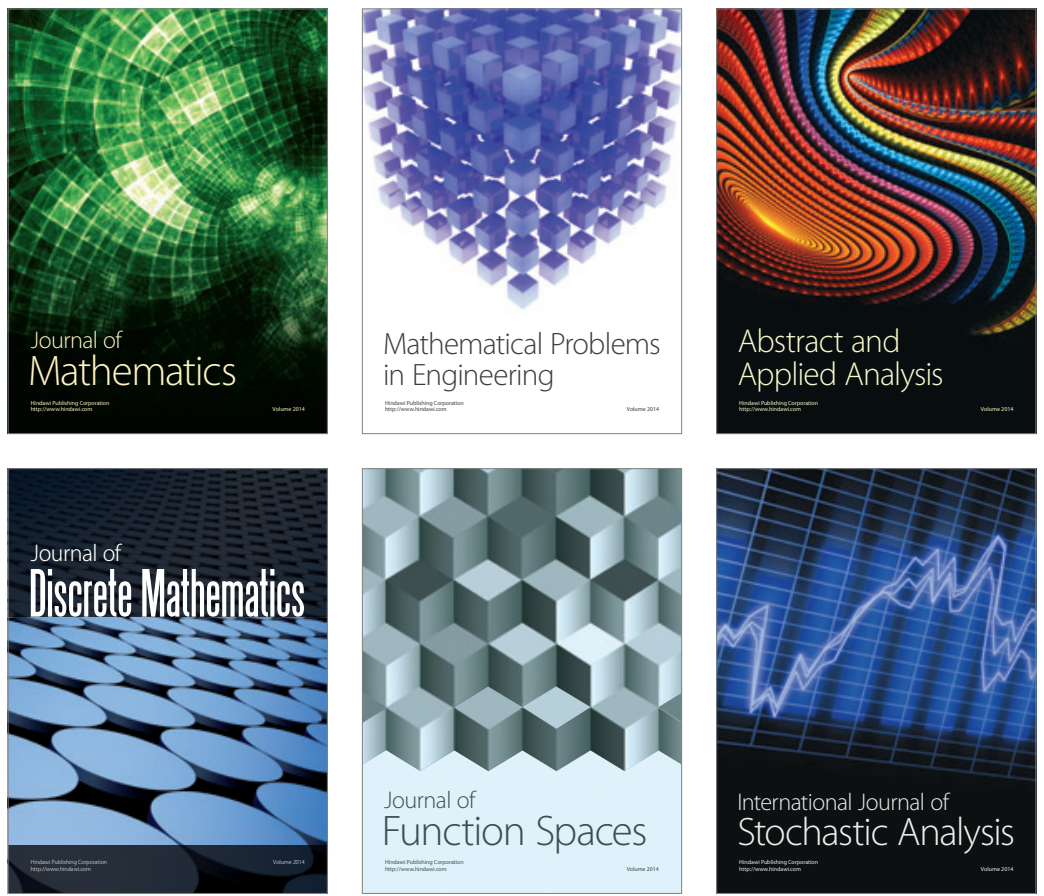

Journal of

Function Spaces

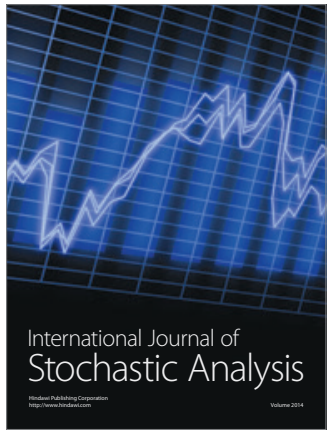

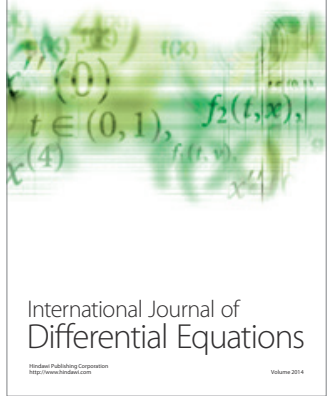
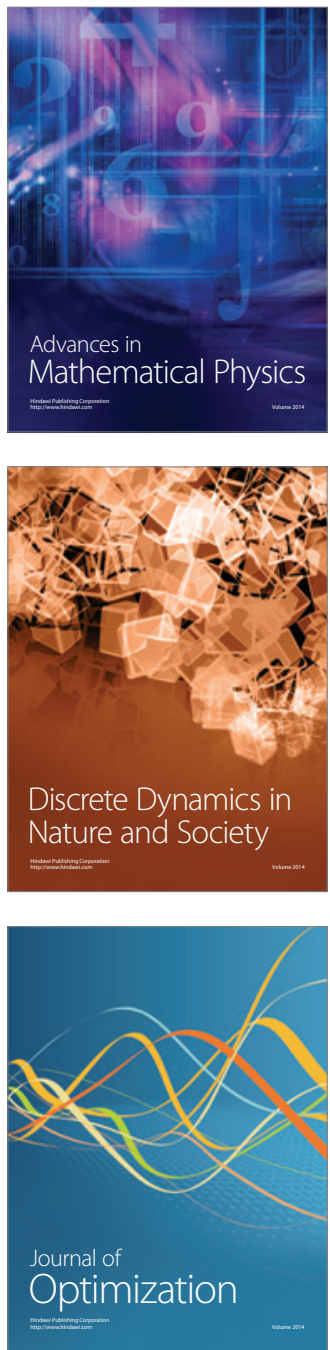